\title{
CLASSICAL TO QUANTUM CROSSOVER OF THE CRITICAL BEHAVIOUR OF IMPURE SYSTEMS
}

\author{
I. P. Takov \\ CPCM Laboratory, G. Nadjakov Institute of Solid State Physics, \\ Bulgarian Academy of Sciences, BG-1784 Sofia, Bulgaria \\ (Received April 30, 1999; received in final form August 26, 1999)
}

\begin{abstract}
The classical-to-quantum dimensional crossover in a wide class of systems with quenched impurities and inhomogeneities is investigated within the framework of the renormalization group. The one-loop renormalization group equations are derived with the help of a double $(\epsilon, \delta)$-expansion, where the small parameter $\delta$ describes the quantum effects and $\epsilon=2 \sigma-d$ is the usual expansion parameter $(0<\sigma \leq 2 ; d$ is the spatial dimensionality). The classical-to-quantum crossover phenomenon is established and the critical exponents are calculated to first order in $\epsilon$ and $\delta$. The analogy between this approach and that of double $(\epsilon, \delta)$-expansion in treating the extended impurities problem is discussed.
\end{abstract}

Key words: quantum critical behaviour, renormalization group, disorder effects, quantum fluctuations.

PACS number(s): 05.70.Jk, 64.60.Ak, 64.60.Fr

\section{INTRODUCTION}

The influence of the quantum fluctuations on the critical behaviour at extremely low temperatures was established in early pioneering works of Rechester [1], Pfeuty and Elliott [2], Young [3], and Hertz [4]. In particular, the classical-to-quantum dimensional crossover (CQC) of the critical behaviour at zero temperature was discovered [2,3] and comprehensively described for a number of systems [4]. The CQC consists of a change of the spatial dimensionality $d$ to an effective dimensionality $D=(d+z)$, where $z$ is the dynamical critical exponent $[4,5]$. For any temperature $T>0$, the asymptotic critical behaviour is classical, whereas for $T \rightarrow 0$, the width of the classical Ginzburg region (see, e.g., Ref. [5]) tends to zero, and at $T=0$ the critical behaviour is entirely a quantum phenomenon. According to $\mathrm{CQC}$ [2-4], the quantum critical behaviour at $T=0$ in a $d$-dimensional quantum system is equivalent to the usual critical behaviour [5] in a $D=(d+z)$-dimensional classical system. An exception is the quantum critical behaviour of interacting real bosons [6].

The effect of quenched disorder of randomly distributed quenched impurities (and/or inhomogeneities) $[7,8]$ on the quantum critical behaviour at $T=0$ of a wide class of quantum models was investigated by Korutcheva and Uzunov [9]; see also Ref. [10]. In contrast to pure systems, where the CQC produces a stable zerotemperature (quantum) critical behaviour [4], standard renormalization group (RG) studies $[9,10]$ performed within the usual $\epsilon$-expansion have shown that the zerotemperature critical behaviour in disordered systems exhibits $[9,10]$ an instability with respect to the quenched disorder. This disorder-induced instability has been deduced from the lack of stable fixed points (FPs) of the RG equations for spatial dimensionalities $d<d_{U}$, where $d_{U}$ is the upper borderline dimensionality. An attempt of a treatment of this instability in particular models was made [11-13] with the help of double $\epsilon$-expansions known from the problem for extended impurities $[14,15]$. It has been claimed [14, 15] on the basis of these double $\epsilon$-expansions that a stable quantum critical behaviour in disordered systems may occur and this point requires a special attention. Reviews of the present status of the theory of quantum critical phenomena in pure and impure systems have been recently given in Refs. [5, 16-18].

In this paper we shall investigate the quantum critical behaviour of impure systems with the help of the renormalization group equations in the one-loop approximation. By using the double $(\epsilon, \delta)$-expansion previously introduced for thin films $[19,20]$, which is quite similar to the original Dorogovtsev double $\epsilon$-expansion [14], we shall demonstrate a special form of CQC for a wide class of effective Bose models of systems with short-range random impurities with a Gaussian distribution. The dynamical and static critical exponents will be calculated to first order in $\epsilon$ and $\delta$. The stability of RG FPs will be discussed and a stable quantum critical behaviour will be revealed.

In Section II we shortly represent the models of consideration. In Section III we generalize the RG analysis of Korutcheva and Uzunov [9] with the help of a double $(\epsilon, \delta)$-expansion within the framework of the one-loop RG approximation. For our aims we shall apply the socalled $\delta$-integration $[19,20]$. In Section IV we summarize and discuss our results.

\section{MODEL}

We shall use the Hamiltonian $\mathcal{H}\left(=-H / T, k_{B}=1\right)$ of the general form [9]

$$
\mathcal{H}=\mathcal{H}_{0}+\mathcal{H}_{4}+\mathcal{H}_{i}
$$

where 


$$
\mathcal{H}_{0}=\sum_{\alpha, q}[f(q)-r] \psi_{\alpha}^{*}(q) \psi_{\alpha}(q)
$$

is the free part,

$$
\mathcal{H}_{4}=-\frac{u T}{2 V} \sum_{\alpha \beta ; q_{1}, q_{2}, q_{3}} \psi_{\alpha}^{*}\left(q_{1}\right) \psi_{\beta}^{*}\left(q_{2}\right) \psi_{\alpha}\left(q_{3}\right) \psi_{\beta}\left(q_{1}+q_{2}-q_{3}\right)
$$

is the usual $\psi^{4}$-interaction term, and

$$
\mathcal{H}_{i}=-V^{-1 / 2} \sum_{\alpha ; \omega_{l} ; \mathbf{k}_{1}, \mathbf{k}_{2}} \phi\left(\mathbf{k}_{1}-\mathbf{k}_{2}\right) \psi_{\alpha}^{*}\left(\omega_{l}, \mathbf{k}_{1}\right) \psi_{\alpha}\left(\omega_{l}, \mathbf{k}_{2}\right)
$$

is the term describing quenched impurities. In Eqs. (2)(4), $\psi(q)=\left\{\psi_{\alpha}, \alpha=1, \ldots, n / 2\right\}$ is the complex fluctuation (order parameter) field which depends on the frequency-momentum vector $q=\left(\omega_{l}, \mathbf{k}\right)$, where $\omega_{l}=$ $2 \pi l T$ with $\hbar=1$ and $l=(0, \pm 1, \ldots)$ is the (Bose) Matsubara frequency, and $\mathbf{k}=\left\{k_{i}=2 \pi n_{i} / L_{i}, n_{i}=0, \pm 1, ..\right\}$ is the $d$-dimensional wave vector. The volume $V$ of the $d$-dimensional system is $V=\left(L_{1} \ldots L_{d}\right), r=r_{0}\left(T-T_{c}\right)$, where $T_{c} \geq 0$ is the bare (unrenormalized) critical temperature, and $u>0$ is the interaction constant.

As the Hamiltonian $\mathcal{H}$ given by Eqs. (1)-(4) is supposed to describe quasimacroscopic quantum and classical fluctuations we shall consider small wave vectors $\mathbf{k}$, i.e., we shall apply the long-wavelength approximation $k a \ll 1$, where $k=|\mathbf{k}|$ and $a$ is the lattice constant (or, the mean interparticle distance in noncrystalline bodies). Thus we shall conveniently choose an upper momentum cutoff $\Lambda$, so that $0<k \leq \Lambda \ll(\pi / a)$. By a suitable choice of the units we set $\Lambda=1$. The frequency $\omega_{l}$ has no natural cutoff but the latter can be always introduced $\left(\left|\omega_{l}\right|<\Lambda_{\omega}\right)$. Note, that only the small frequency $\left(\left|\omega_{l}\right| \sim 0\right)$ behaviour of the modes $\psi(q)$ is relevant to the critical behaviour in both classical $\left(\omega_{l} \equiv 0\right)$ and quantum $\left(T \rightarrow 0, \omega_{l} \neq 0\right)$ regimes. We shall use the cutoff $\Lambda_{\omega}$ only in cases where the frequency integrals exhibit an unessential ultraviolet divergence in the large frequency limit $\left(\omega_{l} \rightarrow \infty\right)$

In Eq. (2),

$$
f(q)=-\frac{\left|\omega_{l}\right|^{m}}{k^{m^{\prime}}}-k^{\sigma}
$$

where $m, m^{\prime}$ and $\sigma$ are positive exponents. Eq. (5) represents a general form of the function $f(q)$ which stands for a number of effective Bose models of real sysems; see, e.g., Refs. [4, 5, 9, 16]. The exponent $\sigma=2$ corresponds to short-range forces whereas $0<\sigma<2$ corresponds to long-range interactions (see, e.g., Refs. [5, 21, 22]). The values of the exponents $m$ and $m^{\prime}$ depend on the particular systems $[4,5,16]$, for example, itinerant antiferromagnets $\left(m=1, m^{\prime} \geq 0\right)$, superconductors $\left(m=1, m^{\prime}=0\right)$, clean itinerant ferromagnets $\left(m=m^{\prime}=1\right)$, dirty itin- erant ferromagnets ( $m=1, m^{\prime}=2$ ), exciton systems $\left(m=1\right.$ or $\left.2, m^{\prime}=0\right)$. The form $f(q)=-\left(i \omega_{l}-k^{2}\right)$ which also describes a number of interesting systems $[5$, 16], in particular, dilute Bose gases, will not be considered in this paper. The last class of systems exhibits a quite unusual quantum critical behaviour as a result of a great simplification of the RG equations [6] in the limit $T \rightarrow 0$; see also the review $[5,17]$.

In Eq. (4), $\phi(\mathbf{k})$ is a random function which obeys the Gaussian distribution $[\ldots]_{R}$ given by

$$
\left[\phi^{*}(\mathbf{k}) \phi\left(\mathbf{k}^{\prime}\right)\right]_{R}=\Delta \delta_{\mathbf{k}, \mathbf{k}^{\prime}}
$$

where $\Delta$ is a parameter describing the disorder effects (short-range random correlations [5, 7-9]). The bare (unrenormalized) Green function $G_{0}=-\left\langle\left|\psi_{\alpha}(q)\right|^{2}\right\rangle$ is given by

$$
G_{0}(q)=\frac{1}{f(q)-r}
$$

\section{RG ANALYSIS}

\section{A. RG equations}

The Wilson-Fisher RG recursion relations [9] can be derived in a variant of the initial rescaling procedure for which the frequency $\omega_{l}$ is not scaled, the initial scaling of the wave vector is given by $\mathbf{k}=\mathbf{k}^{\prime} b^{-1}$, where $b>1$ is the rescaling number, and the field components $\psi_{\alpha}(q)$ obey the scaling relation $\psi_{\alpha}\left(\omega_{l}, \mathbf{k} b^{-1}\right)=\zeta(b) \psi_{\alpha}\left(\omega_{l}, \mathbf{k}\right)$. The RG recursion relations to one-loop approximation can be written in the general form [9]

$$
\begin{aligned}
f^{\prime}(q) & =\zeta^{2}(b)\left[f\left(\omega_{l}, k b^{-1}\right)+\Delta K\left(\omega_{l}\right)\right] \\
r^{\prime} & =\zeta^{2}(b)\left[r+\frac{(n+2)}{2} u I_{1}(r)-\Delta J_{1}(r)\right] \\
(u T)^{\prime} & =\zeta^{4}(b) b^{-d}\left[u T-\frac{(n+8)}{2} u^{2} T I_{2}(r)\right. \\
& \left.+6 \Delta(u T) J_{2}(r)\right] \\
\Delta^{\prime} & =\zeta^{4}(b) b^{-d}\left[\Delta+4 \Delta^{2} J_{2}(r)-(n+2) \Delta u I_{2}(r)\right]
\end{aligned}
$$

where

$$
K\left(\omega_{l}\right)=\int^{\prime} \frac{d^{d} k}{(2 \pi)^{d}}\left\{[f(0, k)-r]^{-1}-\left[f\left(\omega_{l}, k\right)-r\right]^{-1}\right\}
$$




$$
\begin{aligned}
& I_{1}(r)=-T \sum_{\omega_{l}} \int^{\prime} \frac{d^{d} k}{(2 \pi)^{d}} G_{0}(q), \\
& I_{2}(r)=-\frac{\partial I_{1}(r)}{\partial r}=T \sum_{\omega_{l}} \int^{\prime} \frac{d^{d} k}{(2 \pi)^{d}} G_{0}^{2}(q),
\end{aligned}
$$

and

$$
J_{i}(r)=\int^{\prime} \frac{d^{d} k}{(2 \pi)^{d}}\left(k^{\sigma}+r\right)^{-i}, \quad i=1,2 .
$$

The prime $(')$ in the integrals denotes an integration in the shell $b^{-1}<k<1(\Lambda=1)$. The scaling factor $\zeta(b)$ is chosen in the form $\zeta(b)=b^{1-\eta / 2}$, where $\eta / 2=(1-\sigma / 2)$ is the anomalous dimension of the field $\psi_{\alpha}(q)$. Note, that the relation $(\eta / 2)=1-(\sigma / 2)$ is exact $[21,22]$ for all $0<\sigma<2$ (long-range interactions), and the result $\eta=0$ for $\sigma=2$ is valid only in the one-loop order of the theory considered here. The sums (13) and (14) over $\omega_{l}$ are divergent for $m \leq 1$. In order to include values of $m \in(0,1]$ we must introduce an upper frequency cutoff $\Lambda_{\omega}$. The latter is irrelevant to models where $m>1$. For $m<1$, the finite cutoff $\Lambda_{\omega}$ leads to a modification of the RG analysis but the final results for $m>1$ and $0<m<1$ are the same.

The analysis [9] of the RG recursion relations (8)-(11) has been performed within the $\epsilon=(2 \sigma-d)$-expansion in two limiting cases:

(i) The classical case $\left(\lambda_{T} \ll \xi\right)$ when the de Broglie thermal wavelength $\lambda_{T}=\lambda_{T}^{(0)} / T^{\left(1 / z_{0}\right)}$ described by the mean field value $z_{0}$ of the dynamical critical exponent $z$ [17] is much smaller than the (bare) correlation length $\xi=\xi_{0} /\left|T-T_{c}\right|^{1 / \sigma} ; \Lambda_{T}^{(0)}$ and $\xi_{0}$ are scaling amplitudes.

(ii) The quantum case $\left(\lambda_{T} \gg \xi\right)$ which is possible for $T_{c}=0$ and $T \rightarrow 0$.

The analysis of the classical asymptote (i) yields the usual classical critical behaviour governed by the classical fluctuation modes $\psi_{\alpha}(0, \mathbf{k})$. In addition to this trivial result one reveals [9] the critical dynamics of the systems which results from purely quantum dynamical phenomena (for the corresponding values of the dynamical critical exponent $z$ calculated to one-loop order for both cases (i) and (ii), see Ref. [9]; for calculations of $z$ in particular cases, see Refs. [10-13]).

It has been for a first time demonstrated in Ref. [9], that the asymptote (ii) reveals a special type of instability of the quantum critical behaviour at $T=0$. In contrast to pure systems ( $\Delta=0$, Ref. [4]), where CQC $(d \rightarrow d+z)$ manifests itself, the quenched disorder $(\Delta>0)$ in impure systems enhances the classical fluctuations up to an upper borderline dimensionality $d_{U}=2 \sigma$ and CQC cannot occur [9]. The same instability was noted and extensively investigated in more recent works [10, 11-13].

The summation over the frequency $\omega_{l}$ in Eqs. (13) and (14) yields transcendental functions which should be further integrated over the wave vector $\mathbf{k}$. The presence of transcedental functions in Eqs. (8)-(11) leads to a great complication of the RG analysis in the general case of any ratio $0<\left(\lambda_{T} / \xi\right)<\infty$. In result the standard analysis [9] can be performed with analytical means only in the asymptotic cases (i) and (ii). Therefore the entire investigation of the problem requires another approach [19, $20]$, which will be shortly introduced in the next Section.

\section{B. RG equations from a $\delta$-integration}

In the remainder of this paper we shall present another variant of the RG equations (8)-(11) by using a double $(\epsilon, \bar{\delta})$-expansion, where $\bar{\delta}=z_{0} \delta ; z_{0}=\left(\sigma+m^{\prime}\right) / m$ [4] is the mean field value of the dynamical exponent $z$, and $0 \leq \delta \leq 1$. Furthermore, we shall perform the main steps of the RG analysis in terms of the small parameters $\epsilon=(2 \sigma-d)$ and $\bar{\delta}$. In fact, the derivation of RG equations can be performed with the help of a single expansion parameter $\tilde{\epsilon}=2 \sigma-d-\bar{\delta}$, related to an effective upper dimensionality $d_{\text {eff }}=2 \sigma-\bar{\delta}$ [20]. But at a next stage of consideration, when we impose the requirement for a scaling invariant form of the RG equations the single $\tilde{\epsilon}$-expansion should be split to the double $(\epsilon, \bar{\delta})$-expansion.

The quantum effects will be taken into account by the $\bar{\delta}$-dependence of the relevant physical quantities. An attempt along this direction of research has been made for the particular cases of Bose systems [11, 12], with a $\omega_{l}$-dependence of type $f\left(\omega_{l}, 0\right)=-i \omega_{l}$, and for impure quantum antiferromagnets [13], where $f\left(\omega_{l}, 0\right)=\left|\omega_{l}\right|$. We shall follow the method of $\delta$-integration presented in Ref. [20] applied to thin films.

The main idea of the $\delta$-integration $[19,20]$ is to change the usual rule

$$
T \sum_{\omega_{l}} \stackrel{T \rightarrow 0}{\rightarrow} \int_{0}^{\infty} \frac{d \omega}{2 \pi}
$$

by

$$
T \sum_{\omega_{l}} \rightarrow T^{1-\delta} \int \frac{d^{\delta} \omega}{(2 \pi)^{\delta}}
$$

Advantages and disadvantages of the approximate rule (17) were widely discussed in Ref. [20]. In particular, it has been shown that for all intermediate cases $0<\delta<1$, the $\delta$ - integration gives incorrect values of $\mathrm{FP}$ coordinates but universal quantities such as the critical exponents can be reliably calculated. In the limiting cases $\delta \rightarrow 0$ (classical) and ( $\delta \rightarrow 1$ ) (quantum), one obtains an entirely correct description. For models corresponding to $m \leq 1$ one should introduce in Eqs. (16)-(17) an upper frequency cutoff $\Lambda_{\omega}<\infty$. Below we shall consider the case $m>1$ where the finite cutoff $\Lambda_{\omega}$ is not necessary. The case $m \leq 1$ will be briefly discussed in Section IV.

Using the rule (17) in Eqs. (13) and (14) and performing certain standard calculations, the Eqs. (8)-(11) yield 


$$
\begin{gathered}
\omega_{l}^{\prime}=b^{z_{0}} \omega_{l}\left[1+\frac{1}{m} \delta_{m^{\prime}, 0} \mu\left(\frac{b^{\bar{\delta}}-1}{\bar{\delta}}\right)\right] \\
r^{\prime}=b^{\sigma}\left\{r+\frac{(n+2)}{2} v\left[\frac{1-b^{-\sigma}}{\sigma}\left(\frac{m}{m-\delta}\right)-r \ln b\right]\right. \\
\left.-\mu\left[\frac{1-b^{-\sigma+\bar{\delta}}}{\sigma-\bar{\delta}}-r\left(\frac{b^{\bar{\delta}}-1}{\bar{\delta}}\right)\right]\right\}
\end{gathered}
$$$$
v^{\prime}=b^{2 \sigma-d}
$$$$
\times\left(\frac{T}{T^{\prime}}\right)^{\delta}\left\{v-\frac{(n+8)}{2} v^{2} \ln b+6 \mu v\left(\frac{b^{\bar{\delta}}-1}{\bar{\delta}}\right)\right\},
$$

and

$$
\mu^{\prime}=b^{2 \sigma-d}\left\{\mu-(n+2) \mu v \ln \mathrm{b}+4 \mu^{2}\left(\frac{b^{\bar{\delta}}-1}{\bar{\delta}}\right)\right\}
$$

where $\mu=K_{d} \Delta$ with $K_{d}=2^{1-d} \pi^{-d / 2} / \Gamma(d / 2)$, and $v=T^{1-\delta} a u$ with $a=K_{d} K_{\delta} \Gamma(\delta / m) \Gamma(2-\delta / m) / m$. The Eqs. (18)-(21) are valid within an $\tilde{\epsilon}=(2 \sigma-d-\bar{\delta})$ expansion, where the perturbation integrals are calculated at the upper borderline dimensionality $d_{U}=(2 \sigma-\bar{\delta})$. It is obvious that Eqs. (18)-(21) take a scaling invariant form provided we assume that $\left(b^{\bar{\delta}}-1\right) \approx \bar{\delta} \ln b$, i.e., for $\bar{\delta} \ll 1$. This means that we must substitute the single expansion parameter $\tilde{\epsilon}$ with two ones: $\epsilon=(2 \sigma-d)$ and $\bar{\delta}$.

Eq. (18) for $\omega_{l}$ can be written for the temperature:

$$
T^{\prime}=b^{z(\mu)} T
$$

where

$$
z(\mu)=z_{0}+\frac{\mu}{m} \delta_{m^{\prime}, 0} .
$$

The FP value $\mu^{*}$ of $\mu$ yields the dynamical critical exponent: $z=z\left(\mu^{*}\right)$. Obviously, the mean field value $z_{0}=\left(\sigma+m^{\prime}\right) / m$ will acquire $(\epsilon, \delta)$-corrections only for $\mu^{*} \neq 0$, i.e., for random FPs (RFP). At the classical asymptote $(\delta \rightarrow 0)$, the dynamical exponent $z\left(\mu^{*}\right)$ was calculated in Ref. [9]. Here the calculation can be generalized for $\delta>0$.

It is convenient to perform our further analysis with the help of the corresponding differential RG equations (see, e.g., Ref. [5, 11-13]). From Eqs. (19)-(23) one can easily obtain

$$
\frac{d T(l)}{d l}=z(\mu) T(l)
$$

$$
\begin{aligned}
\frac{d r(l)}{d l} & =\sigma r(l)+\frac{(n+2)}{2} v(l)\left[\frac{m}{m-\delta}-r(l)\right] \\
& -\mu(l)[1-r(l)]
\end{aligned}
$$

$$
\begin{aligned}
\frac{d v(l)}{d l} & =[2 \sigma-d-\delta z(\mu)] v(l) \\
& -\frac{(n+8)}{2} v(l)^{2}+6 v(l) \mu(l),
\end{aligned}
$$

$$
\frac{d \mu(l)}{d l}=(2 \sigma-d) \mu(l)-(n+2) v(l) \mu(l)+4 \mu(l)^{2},
$$

In Eq. (26) one can safely ignore a small term $\delta \mu v \sim \delta \epsilon^{2}$ and substitute $z(\mu)$ with $z_{0}$.

\section{Fixed points and critical exponents}

Since $z>0$, Eq. (24) has two FPs: $T_{C}^{*}=\infty$ (classical) and $T_{Q}^{*}=0$ (quantum). Within the present $\mathrm{RG}$ scheme the classical FP $\left(T_{C}^{*}=\infty\right)$ corresponds to the classical critical behaviour at $T>0$, where the frequencies $\omega_{l}$ are irrelevant to the asymptotic (critical) thermodynamics at $T \rightarrow T_{c}>0$. In this case, Eq. (18) indicates that the successive RG transformations lead to $\omega_{l} \rightarrow \infty$ for all $\omega_{l}>0$. Thus the perturbation contributions from the noncritical modes $\psi_{\alpha}\left(\omega_{l}>0, \mathbf{k}\right)$ become irrelevant and the consequences of this circumstance is described by FPs of type $T_{C}^{*}=\infty$. Within our approach this classical critical behaviour is described by Eqs. (18)-(21) or, equivalently, by Eqs. (24)-(27) in the limit $\delta \rightarrow 0$. The quantum FPs $\left(T_{Q}^{*}=0\right)$ correspond to the limit $\delta \rightarrow 1$. The Eqs. (24) and (25) constitute $T$ and $r$ as the relevant thermodynamic parameters.

The Eqs. (26)-(27) give four FPs:

(1) Gaussian (GFP)

$$
v_{G}=\mu_{G}=0,
$$

(2) Unphysical (UFP)

$$
v_{U}=0, \mu_{U}=-\frac{\epsilon}{4}
$$

(3) Heisenberg (HFP)

$$
v_{H}=\frac{2(\epsilon-\bar{\delta})}{(n+8)}, \quad \mu_{H}=0
$$

(4) Random (RFP) 


$$
v_{R}=\frac{\epsilon+2 \bar{\delta}}{2(n-1)} \quad, \quad \mu_{R}=\frac{(4-n) \epsilon+2(n+2) \bar{\delta}}{8(n-1)}
$$

The Eqs. (31) are valid for $n>1$. For $\bar{\delta}=0$, all these FPs are known from Refs. [7, 9]. In some cases the FPs (1)-(4) will be shortly referred to as $G, U, H$ and $R$, respectively. Here we shall consider the quantum effects represented by the $\bar{\delta}$-dependence of the FPs and related physical quantities.

The Eq. (6) defines $\mu=K_{d} \Delta$ as a positive parameter and, hence, the UFP does not present a practical interest for $\epsilon>0$; for $\epsilon<0$ UFP is physical but unstable $[7,9]$. For $2 \sigma<d<2(\sigma+\bar{\delta})$ the UFP has an instability towards $\mu$ whereas the same FP has a double instability (towards both $v$ and $\mu$ ) for $d>2(\sigma+\bar{\delta})$. We shall consider $G, H$, and $R$ in more details.

The stability exponents of GFP are $\lambda_{v}^{(G)}=\epsilon-\bar{\delta}$ and $\lambda_{\mu}^{(G)}=\epsilon$. Thus GFP is stable only for $d>2 \sigma$. The stability exponents of $H$ are

$$
\lambda_{v}^{(H)}=\bar{\delta}-\epsilon
$$

and

$$
\lambda_{\mu}^{(H)}=\frac{(4-n) \epsilon+2(n+2) \bar{\delta}}{(n+8)} .
$$

The stability exponents $\lambda_{1,2}^{(R)}$ of the RFP are given by

$$
\lambda_{1,2}^{(R)}=-\frac{3 n \epsilon+2(4-n) \bar{\delta} \mp \sqrt{Q}}{8(n-1)}
$$

where

$$
\begin{aligned}
Q & =(5 n-8)^{2} \epsilon^{2}-12\left(5 n^{2}+8 n-16\right) \bar{\delta}^{2} \\
& -12\left(n^{2}+12 n-16\right) \epsilon \bar{\delta}
\end{aligned}
$$

For $Q \geq 0$, the correction-to-scaling (stability) exponents $\lambda_{1,2}^{(R)}$ take real values. The usual (impure) critical behaviour [7] is stable for $\lambda_{1,2}^{(R)}<0$. For $Q<0$ the complex exponents $\lambda_{1,2}^{(R)}$ describe the so-called impure critical behaviour with oscillatory corrections to the main scaling laws $[8,14]$. The corresponding RFP is often referred to as a "focal" FP. We shall denote the RFP in such cases by $\mathrm{R}_{C} \mathrm{FP}$ or simply by $R_{C}$. The RFP with real values of $\lambda_{1,2}^{(R)}$ will be respectively referred to as $\mathrm{R}_{R} \mathrm{FP}$ or, simply, as $R_{R}$. The same notations will be used for respective quantities (critical exponents, stability domains).

The static critical behaviour is given by $\eta=(2-\sigma)$ and the value of the correlation length exponent $\nu$. For $G, \nu_{G}=1 / \sigma$. For $H$ we obtain

$$
\nu_{H}=\frac{1}{\sigma}+\frac{(n+2)(\epsilon-\bar{\delta})}{\sigma^{2}(n+8)}
$$

and for $R$ we have

$$
\nu_{R}=\frac{1}{\sigma}+\frac{3 \epsilon n+2(n+2) \bar{\delta}}{8 \sigma^{2}(n-1)}, \quad n>1 .
$$

The dynamical exponent $z$ is $z=z_{0}=\left(\sigma+m^{\prime}\right) / m$ for GFP and HFP. For $R$ the dynamic exponent is given by

$$
z_{R}=\frac{\sigma}{m}+\frac{1}{m}\left[\frac{(4-n) \epsilon+2(n+2) \bar{\delta}}{8(n-1)}\right], \quad n>1
$$

for $m^{\prime}=0$, and $z_{R}=z_{0}$ for $m^{\prime}>0$. The Eq. (38) is a generalization of the result for $z$ obtained in Ref. [9] for $\delta=0$. Besides, by setting $m=1$ and $\sigma=2$ in Eq. (38) one obtains the result for $z_{R}$ known from Ref. [13]. For $\sigma=2, m=2$ and $\bar{\delta} \equiv \delta\left(z_{0}=1\right), z_{R}$ from Eq. (38) reproduces the result for the critical exponent $z_{R}$ obtained in Ref. [20].

\section{Stability analysis}

Here we shall briefly discuss the stability of $H$ and $R$ for $n>1$. Besides, we shall distinguish between the critical behaviour described by real $(\mathrm{R})$ and complex $(\mathrm{C})$ values of the correction-to-scaling exponents $\lambda_{1,2}^{(R)}$.

The stability requirements for $H$ are: $\lambda_{v}^{(H)}<0$, and $\lambda_{\mu}^{(H)}<0$. To these inequalities we must add the restriction $d_{L}=(\sigma-\bar{\delta})<d$ of our RG analysis $\left(d_{L}-\right.$ the lower borderline dimension). For $\epsilon<0, H$ is unstable towards $v$-perturbation for all $n>0$ and $\bar{\delta} \geq 0$. For $\epsilon>0$ one can easily demonstrate that $H$ is unstable for all $0<n \leq 4$ and $0 \leq \bar{\delta} \leq 1$ except for the case $n=4$ for $\bar{\delta}=0$ in which $\bar{H}$ has a known marginal stability [7]. For $n>4$, $H$ is stable provided

$$
d_{L}=(\sigma-\bar{\delta})<d<d_{H}
$$

where

$$
d_{H}=2 \sigma-\frac{2(n+2)}{(n-4)} \bar{\delta}
$$

The HFP has a domain of stability in the plane, say, $(d, \delta)$ provided $0<d_{L}<d_{H}$. From (39)-(40) we obtain

$$
\kappa \delta<\frac{(n-4)}{(n+8)}
$$

where $\kappa=\left(z_{0} / \sigma\right)$. For $(\kappa \delta)=0$ (classical limit) the condition (41) is satisfied for any $n>4$.

The inequality (41) can be solved with respect to $n$ to first order in the $\delta$-expansion: 


$$
\begin{gathered}
n>n_{H}(\delta), \\
n_{H}(\delta)=(4+12 \kappa \delta) .
\end{gathered}
$$

The condition $d_{L}>0$ yields $(\kappa \delta)<1$. Besides the $(\kappa \delta)$-term in Eq.(42) should be considered small with respect to $n_{H}(0)=4$. This is consistent both with our $\delta$-expansion and the requirement $d_{L} \geq 0$ which leads to $(\kappa \delta)<1$. For $\kappa<1$ the result $(43)$ for $n_{H}(\delta)$ is reliable for a wide range of values of $\delta$. In general, the critical value $n_{H}(\delta)$ will contain higher order corrections in powers of $(\kappa \delta)$ but their calculation is beyond our one-loop RG treatment.

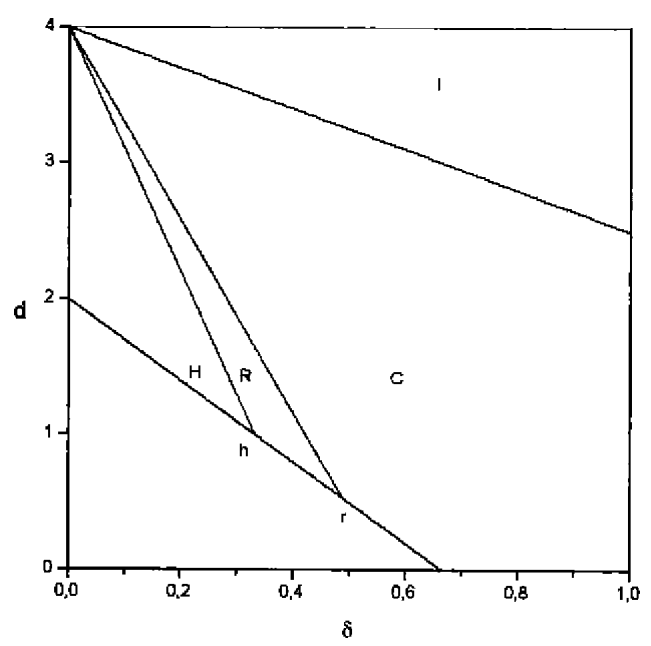

Fig. 1. Stability domains of $H, R_{R}$ and $R_{C}$ for $\kappa=1.5$ and $n=16$.

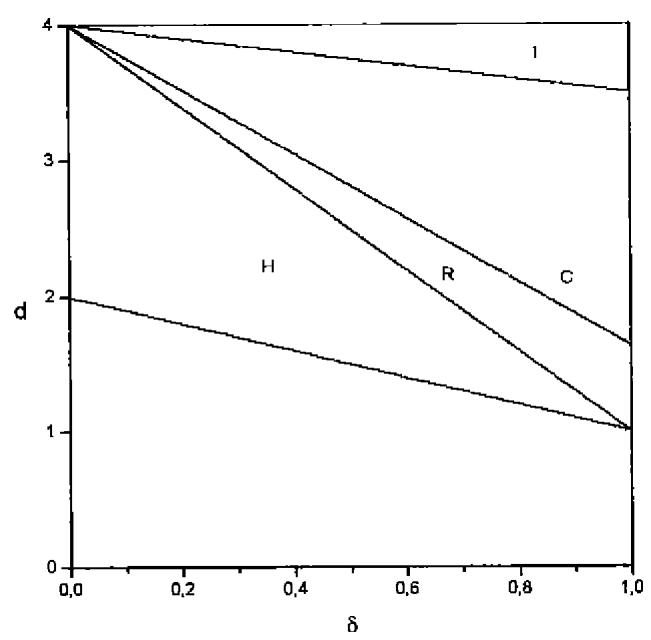

Fig. 2. Stability domains of $H, R_{R}$ and $R_{C}$ for $\kappa=0.5$ and $n=16$.

The inequality (41) can be considered with respect to $\delta$. For $\kappa>0$ we have $\delta<\delta_{H}$, where

$$
\delta_{H}=\frac{(n-4)}{(n+8) \kappa} .
$$

The upper bound $\delta_{H}<1$ always exists for $\kappa \geq 1$; see Fig. 1. In this case the values of $\delta$ in the interval $\left[\delta_{H}, 1\right]$ are forbidden. For $0<\kappa<1$ the additional restriction $\delta \leq \delta_{H}$ on the values of $\delta$ takes place only when

$$
n<n_{H}(\kappa)=\frac{4(2 \kappa+1)}{(1-\kappa)} .
$$

The condition (45) should be considered together with $n>4$. The stability domain of $H$ is depicted in Fig. 2 for $\kappa=1 / 2$ and $n=n_{H}(1 / 2)$; and in Fig. 3 for $\kappa=1 / 2$ and $n=22$.

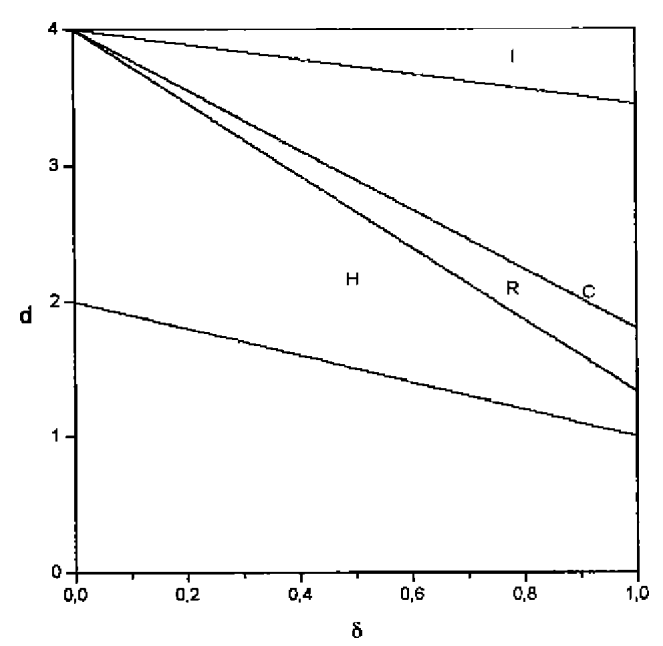

Fig. 3. Stability domains of $H, R_{R}$ and $R_{C}$ for $\kappa=0.5$ and $n=22$.

The stability properties of the RFPs, $R_{R}$ and $R_{C}$, can be investigated only for $n \neq 1$ because of the degeneration of the $\mathrm{RG}$ equations for $n=1$ [7, 8, 14]; for the notations $R_{R}$ and $R_{C}$, see Section III.C. The case $0<n<1$ seems to be of an academic interest only and for this reason we shall focus our attention on symmetry indices $n>1$. We have the following conditions for the stability of $R_{R}$ :

$$
\begin{aligned}
& (\sigma-\bar{\delta})<d<2(\sigma+\bar{\delta}) \\
& (4-n)(2 \sigma-d)+2(n+2) \bar{\delta}>0, \\
& 3 n(2 \sigma-d)+2(4-n) \bar{\delta}>0,
\end{aligned}
$$

and

$$
Q \geq 0
$$

together with $d_{L}=(\sigma-\bar{\delta})>0$. For $R_{C}$ one obtains the inequalities (47), (48), the inequality inverse to (49), and the standard condition $d_{L}>0$. The criteria of stability can be investigated numerically for all values of $d, n>1$, 
and $\delta=\bar{\delta} / z_{0}$. A similar analysis for another problem has been performed in Ref. [20] for values of $n$ of particular interest.

For $n=4, R_{R}$ is stable in the domain $(d, \delta)$ defined by $0<\delta<\delta_{4}$ and $(1-\kappa \delta)<(d / \sigma)<\vec{d}_{R}(4)$, $\delta_{4}=1 /(1+2 \sqrt{3}) \kappa$ and $\bar{d}_{R}(4)=2-2(1+\sqrt{3}) \kappa \delta$. Besides, we should note that $\left(d_{L} / \sigma\right)=(1-\kappa \delta)>0$ must be satisfied throughout our analysis.

For $1<n<4$, the stability of $R_{R}$ is given by $Q \geq 0$ and

$$
1-\kappa \delta<(d / \sigma)<\bar{d}_{R}
$$

with $\bar{d}_{R}=d_{R} / \sigma$, where

$$
d_{R}=2 \sigma+\frac{2(4-n) \bar{\delta}}{3 n}
$$

For $R_{C}$, we must use the inequalities $Q<0$ and (50). The domains of stability of $R_{R}$ and $R_{C}$ are depicted in Figs. 1, 2, and 3. Another special case is $n=8 / 5$ [23]. At $n=8 / 5$ the $\epsilon$-expansion exhibits singularities [23]. In our case of one-loop RG analysis the stability domain of $R_{R}$ at $n=8 / 5$ does not exist at all and the domain of $R_{C}$ is expanded up to $0 \leq \delta \leq 1$ and $(1-\kappa \delta)<(d / \sigma) \leq(2+\kappa \delta)$.

Both $H$ and $R$ are stable for $n>4$ in different domains of the $(d, \delta)$ plane. For $\delta<\delta_{H}$ the stability domain of $R_{R}$ is given by $Q \geq 0$ and

$$
\bar{d}_{H}<(d / \sigma)<\bar{d}_{R}
$$

whereas for $\delta>\delta_{H}$, the same domain is described by the inequalities $Q \geq 0$ and

$$
1-\kappa \delta<(d / \sigma)<\bar{d}_{R}
$$

The stability domain of $R_{C}$ is given by (52) and (53) and $Q<0$. The stability domains $R$ and $C$ of $R_{R}$ and $R_{C}$ are shown in Figs. 1, 2, and 3 for particular values of $\kappa$ and $n$. The borderline separating the stability domains of $R_{R}$ and $R_{C}$ is given by

$$
d / \sigma=2-\rho \delta
$$

where

$$
\rho=\frac{2 \kappa\left[3 n^{2}+36 n-48+8 \sqrt{6(n+2)(n-1)^{3}}\right]}{(5 n-8)^{2}}
$$

In certain cases, such as that in Fig. 1, the borderline (54) crosses the upper borderline of stability region of $H$ at a coordinate $\delta_{R}<1$, which is given by

$$
\delta_{R}=\frac{(5 n-8)^{2}}{\kappa\left[152 n-19 n^{2}-160+16 \sqrt{6(n+2)(n-1)^{3}}\right]} .
$$

For example, if $\kappa=0.5$ and $\sigma=2$, one obtains $\delta_{R}<1$ provided $1.23<n<8.43$.

The domains in Figs. 1-3 denoted by I correspond to a total instability of the system for $n>4$. In these domains all FPs are unstable for all $n>4, \delta>0$ and $d \sim 2 \sigma$. The instability can be interpreted $[13,20]$ as an indication for a fluctuation-driven phase transition of first order.

\section{MAIN RESULTS AND DISCUSSION}

We have derived the one-loop RG equations for a wide class of models of quantum critical phenomena. Our main attention has been focussed on the zero temperature critical phenomena in systems with quenched impurities. For our purposes we have used an $(\epsilon, \delta)$-expansion, where the parameter $0 \leq \delta \leq 1$ describes the quantum effects. The critical exponents corresponding to FPs of the RG equations are calculated to first order in $\epsilon=(2 \sigma-d)$ and $0 \leq \delta \leq 1$. The stability properties of the FPs are investigated. It has been demonstrated within the double $(\epsilon, \delta)$-expansion that several types of stable critical behaviour are possible.

Predictions about the zero temperature (quantum) critical behaviour can be made by the extrapolation of the results for $\delta \ll 1$ to $\delta \sim 1$. The small $(\delta \ll 1)$ corrections to the results known from the usual $\epsilon$-analysis account for quantum effects on the usual classical behaviour at finite-temperature critical points $\left(T_{c} \neq 0\right)$. The variation of the parameter $\delta$ can be considered as dependent on the ratio $\left(\lambda_{T} / \xi\right)[17,20]$. The quantum limiting case $\delta \sim 1$ corresponds to $\left(\lambda_{T} / \xi\right) \sim \infty$ whereas the classical asymptote $\delta \rightarrow 0$ corresponds to $\left(\lambda_{T} / \xi\right) \sim 0$. Our analysis allows a general treatment corresponding to any value $0 \leq \delta \leq 1$. Note, that within the present $R G$ analysis $\delta$ is considered as a small expansion parameter but the results can be extended to finite values of $\delta$ (of order of unity). So, the expansion parameter $\delta$ is treated at the same footing as the usual expansion parameter $\epsilon$.

The RG Eqs. (25)-(27) formally coincide with the RG equations for classical systems with extended $(\delta-$ dimensional) impurities [14], which are randomly distributed in the rest $d$ dimensions of a $D_{\delta}=(d+\delta)$ dimensional system. In our case, the extra $\delta$ dimension is a purely quantum effect due to the imaginary "time" $\tau$ or, equivalently, on the Matsubara frequency $\omega_{l}$ conjugate to $\tau$. In fact the spatial dimensionality $d$ does not change but for any $\delta>0$ the point impurities contained in the initial Hamiltonian (1)-(4) effectively behave like extended quenched impurities of dimensionality $\delta$. Thus the results obtained by Dorogovtsev [14] for classical systems with extended impurities and short-range interactions $(\sigma=2)$ can be easily rederived from Eqs. (25)(27). For this aim one must change our $\epsilon=(2 \sigma-d)$ 
with $\tilde{\epsilon}=(2 \sigma-d-\bar{\delta})$. For example, in terms of $\tilde{\epsilon}$ and $\bar{\delta}$, Eq. (37) reads

$$
\nu_{R}=\frac{1}{\sigma}+\frac{3 n \tilde{\epsilon}+(5 n+4) \bar{\delta}}{8 \sigma^{2}(n-1)}
$$

One can easily check that there is a formal equivalence between the critical exponents of classical systems with extended impurities and systems with point-like quenched impurities of $\delta$-type as given by Eq. (6). The dynamical exponent $z_{R}$ from Eq. (38) corresponds to the so-called longitudinal exponent $\eta_{l}$ in Ref. [14]. Despite of their different physical meaning, these two exponents are related by $z_{R}=(\sigma / m)+\eta_{l}$. One may easily check that $\left(z_{R}-\sigma / m\right)$ from Eq. (38) coincides with the result [14] for $\eta_{l}$ for $\sigma=m=2$ and $m^{\prime}=0$. It can be therefore concluded that the quantum critical behaviour for a wide class of systems is quite similar to that for classical systems with extended impurities. In fact, for $m^{\prime}=0$ and $m=\sigma=2$, there is a total equivalence in the critical properties of classical systems with extended impurities and systems with point impurities.

The limit $\delta \rightarrow 1$ corresponds to $\left(\lambda_{T} / \xi\right) \rightarrow \infty$, i.e., to the quantum limit $T \rightarrow 0$. In this case our RG investigation in terms of $\epsilon$ and $\delta$ leads to stable FPs which describe several types of quantum critical behaviour. For certain values of the symmetry index $n$ this quantum critical behaviour is influenced by the quenched disorder. This picture corresponds again to that known from classical systems with extended (one-dimensional, $\delta=1$ ) quenched impurities. Similar conclusions were obtained in Refs. [12, 13], where particular models have been investigated. We must emphasize that the prediction of the availability of a stable zero temperature critical behaviour in impure systems is a direct consequence of our supposition that an additional $\delta$-expansion can be performed.

The direct application of the RG method to the same zero temperature problem leads to an instability [9] of the quantum critical behaviour with respect to the same quenched impurities. In our present study, the previously known instability [9] appears only in a close vicinity below the upper borderline dimensionality $d_{U}=2 \sigma$. In both cases the instability indicates a fluctuation induced phase transition of first order.

Our analysis leads to a general conclusion, that the standard CQC concept [4] cannot be directly applied to the quantum critical behaviour of impure systems. The quantum critical behaviour at $T=0$ of such systems with quenched point impurities corresponds to the classical critical behaviour of systems with certain extended impurities rather than to classical systems with point impurities at a higher effective dimensionality $(D=d+z)$. In order to maintain the interpretation of our results within a dimensional CQC concept we must establish a correspondence between the quantum critical behaviour and the critical behaviour of certain classical systems with extended impurities although the initial class of quantum models contains point impurities only.

We conclude with several remarks for the validity of our investigation. Our results for $n>1$ can be extended to higher orders in the loop expansion. The study of Ising-like systems $(n=1)$ requires a two-loop approximation $[7,8,14,23]$. It can be supposed that such an investigation will reveal a quantum critical behaviour quite similar to that for classical Ising $(n=1)$ with short range interaction $(\sigma=2)$ and extended impurities [14]. The two-loop RG analysis for long-range interaction $(0<\sigma<2)$ is quite complicated [21, 22] and for this reason our one-loop consideration for $0<\sigma<2$ cannot be straightforwardly extended to the two-loop order.

The RG analysis in Section III has been performed for systems where $m>1$. For $m<1$ an upper frequency cutoff $\Lambda_{\omega}$ should be introduced in order to avoid a runaway (to infinity) of the FPs coordinates. This does not change the results for the critical exponents and the stability properties of FPs. Thus our main conclusions are valid for all $m>0$. The last conclusion is a result of both heuristic arguments and direct RG calculations. The main heuristic argument is that the upper cutoff $\Lambda_{\omega}$ should not change the critical behaviour as the relevant low frequency modes $\psi_{\alpha}\left(\omega_{l}<\Lambda_{\omega}, \mathbf{k}\right)$ are included into consideration. The direct introduction of $\Lambda_{\omega}$ in the calculations lead to another form of the perturbation integrals but the subsequent RG analysis confirms the simple heuristic argument. This modified RG analysis can be easily accomplished and we shall not dwell on this point.

\section{ACKNOWLEDGEMENTS}

I would like to thank Professor Dimo Uzunov for valuable discussions.
[1] A. B. Rechester, Zh. Eksp. Teor. Fiz. 60, 782 (1971) [Sov. Phys. JETP 33, 423].

[2] P. Pfeuty, R. J. Elliott, J. Phys. C 4, 2370 (1971).

[3] A. P. Young, J. Phys. C 8, L309 (1975).

[4] J. A. Hertz, Phys. Rev. B 14, 1165 (1976).

[5] D. I. Uzunov, Introduction to the Theory of Critical Phenomena (World Scientific, Singapore, 1993).

[6] D. I. Uzunov, Phys. Lett. A 87, 11 (1981).

[7] T. C. Lubensky, Phys. Rev. B 11, 3573 (1975).
[8] D. E. Khmelnitskii, Zh. Eksp. Teor. Fiz. 68, 1960 (1975) [Sov. Phys. JETP, 41, 981 (1975)]; Phys. Lett. A 67, 59 (1978).

[9] E. R. Korutcheva, D. I. Uzunov, preprint BAS (Sofia, 1983); Phys. Lett. A 106, 175 (1984).

[10] G. Busiello, L. De Cesare, I. Rabuffo, Phys. Lett. A 102, 41 (1984).

[11] P. B. Weichman, K. Kim, Phys. Rev. B 40, 813 (1989).

[12] A. M. J. Schakel, Phys. Lett. A 224, 287 (1997). 


\title{
I. P. TAKOV
}

[13] T. R. Kirkpatrik, D. Belitz, Phys. Rev. Lett. 76, 2571 (1996); Err. Phys. Rev. Lett. 78, 1197 (1997).

[14] S. N. Dorogovtsev, Phys. Lett. A 76, 169 (1980); Fiz. Tverd. Tela 22, 321, 3658 (1980) [Sov. Phys. Solid State 22 168, 2141 (1980)].

[15] A. Weinrib, B. I. Halperin, Phys. Rev. B 27, 413 (1983).

[16] A. Caramico D'Auria, L. De Cesare, U. Esposito, I. Rabuffo, Physica A 243, 152 (1997).

[17] L. De Cesare, D. I. Uzunov, in Correlations, Coherence and Order, edited by D. V. Shopova, D. I. Uzunov (Kluwer Academic/Plenum Publishers, New York, 1999), p. 29.

[18] A. M. J. Schakel, in Correlations, Coherence and Order, edited by D. V. Shopova, D. I. Uzunov (Kluwer Academic/Plenum Publishers, New York, 1999), p. 295.

[19] L. De Cesare, L. Craco, I. Rabuffo, D. I. Uzunov J. Phys. Stud. 2, 175 (1998); L. De Cesare, I. Rabuffo, D. I. Uzunov J. Phys. Stud. 2, 181 (1998).

[20] L. Craco, L. D. Cesare, I. Rabuffo, I. P. Takov, D. I. Uzunov, Physica A 270, 486 (1999).

[21] M. E. Fisher, S. K. Ma, B. G. Nickel, Phys. Rev. Lett. 29, 917 (1972).

[22] J. Sak, Phys. Rev. B 8, 281 (1973); M. E. Fisher, Rev. Mod. Phys. 46, 597 (1974).

[23] I. D. Lawrie, Y. T. Millev, D. I. Uzunov, J. Phys. A 20, 1599 (1987); (E) 20, 6159 (1987).

\section{КЛАСИЧНО-КВАНТОВИЙ КРОСОВЕР У КРИТИЧНІЙ ПОВЕДІНЩІ ДОМІШКОВИХ СИСТЕМ}

\author{
I. П. Таков \\ Інститут фізики твердого тіла ім. Г. Наднакова, Болгарсъка академія наук, \\ Софіл, BG-1784, Болгаріл
}

\begin{abstract}
Класично-квантовий кросовер за вимірністю в широкому класі систем із замороженими домішками та неоднорідностями досліджений у рамках методу ренормалізаційної групи. Однопетлеві ренормгрупові рівняння виведено за допомогою подвійного $(\epsilon, \delta)$-розвинення, де локальний параметр $\delta$ описує квантові ефекти, а $\epsilon=2 \sigma-d \epsilon$ звичайним параметром розвинення $(0<\sigma \leq 2 ; d-$ вимірність простору). Установлено явище класично-квантового кросоверу та обчислено критичні експоненти в першому порядку за $\epsilon$ та $\delta$. Обговорено аналогї між цим підходом та підходом подвійного $(\epsilon, \delta)$-розвинення при розгля,ті задачі протяжних домішок.
\end{abstract}

